\title{
The Operations Plan for the MErcury Radiometer and Thermal infrared Imaging Spectrometer (MERTIS) on its way to Mercury
}

\author{
A. Maturilli*a, J. Helbert ${ }^{\mathrm{a}}$, M. D’Amore ${ }^{\mathrm{a}}$, I. Varatharajan ${ }^{\mathrm{a}}$, H. Hiesinger ${ }^{\mathrm{b}}$, K. Bauch $^{\mathrm{b}}$ \\ ${ }^{a}$ Institute of Planetary Research, German Aerospace Centre (DLR), 2 Rutherfordstrasse, Berlin, \\ Germany 12249; ' Institute for Planetology, Münster University, 10 Wilhelm-Klemm-Str., Münster, \\ Germany 48149 \\ *alessandro.maturilli@dlr.de; phone +49030 67055313; dlr.de/pf/
}

\begin{abstract}
The MErcury Radiometer and Thermal infrared Imaging Spectrometer (MERTIS) is a highly integrated instrument to study mineralogy and temperature distribution of Mercury's surface in unprecedented quality. MERTIS was proposed in 2003 as payload of the Mercury Planetary Orbiter spacecraft of the joint ESA-JAXA BepiColombo mission. With the planned launch on top of an Ariane 5 in October of 2018, the mission will soon start its 7 years journey to Mercury.

On its way to Mercury, BepiColombo will have 2 flybys of Venus and one of the Earth-Moon system. MERTIS will obtain data during each of these flybys - for Venus the first mid-infrared spectral data since Venera 15 in 1983.

After arrival at Mercury in 2025 MERTIS will globally map the surface composition with a resolution of 500m, and study surface temperature variations providing an insight into the thermo-physical properties of the surface. To achieve this, MERTIS combines a push-broom IR grating spectrometer (TIS) with a radiometer (TIR) sharing the same optics, instrument electronics and in-flight calibration components for the whole wavelength range of 7-14 $\mu \mathrm{m}$ (TIS) and 7-40 $\mu \mathrm{m}$ (TIR), respectively.

Instrument operations in the challenging environment at Mercury with power and data constraints require a sophisticated mapping scheme for the TIS observations, which also has to account for the MERTIS calibration needs. Execution of this scheme creates challenges for the operation of the instruments, data processing, and the creation of map products. Extensive onground testing and rehearsals during the Venus and Earth flybys will ensure flawless performance at Mercury.
\end{abstract}

Keywords: Observation Strategy, Data Volume Management, Mercury, BepiColombo, MERTIS, Remote Sensing, Flyby, IR-Imaging Spectrometer

\section{INTRODUCTION}

Mercury is an extreme of our planetary system. Since its formation, it has been subjected to the highest temperature and has experienced the largest diurnal temperature variation of any object in the Solar System. It is the closest planet to the Sun and has the highest uncompressed density of all planets. Solar tides have influenced its rotational state. Its surface has been altered during the initial cooling phase and its chemical composition may have been modified by bombardment in its early history. Mercury therefore plays an important role in constraining and testing dynamical and compositional theories of planetary system formation. Besides ground based observations only two American probes have returned data from Mercury: Mariner 10, which made three flybys of Mercury in 1974-1975; obtaining images of somewhat less than half of the planet's surface and discovering its unexpected magnetic field which is however about hundred times weaker than the Earth magnetic field; and MESSENGER, which was launched in 2004, performed 3 Mercury flyby's in 2008 and 2009, started orbiting the planet in March 2011 and ended on 30 April 2015 with a crash on the surface. MESSENGER has already provided many surprising results questioning our current understanding of Mercury's evolution history. BepiColombo and its comprehensive payload is well suited to answer most of the old and new questions raised [1]. The main scientific objectives of the BepiColombo mission are:

- Investigation of the origin and evolution of a planet close to its parent star.

- Investigation of Mercury's figure, interior structure, and composition. 
- Investigation of the interior dynamics and origin of its magnetic field.

- Investigation of the exogenic and endogenic surface modifications, cratering, tectonics, and volcanism.

- Investigation of the composition, origin and dynamics of Mercury's exosphere and polar deposits.

- Investigation of the structure and dynamics of Mercury's magnetosphere.

- Test of Einstein's theory of general relativity.

BepiColombo is the planetary mission of the Cosmic Vision Program of the European Space Agency (ESA). The mission is devoted to the thorough exploration of Mercury and its environment with the aim to understand the process of planetary formation and evolution in the hottest part of the proto-planetary nebula as well as to understand similarities and differences between the magnetospheres of Mercury and Earth. The mission will be carried out as a joint project between ESA and JAXA (Japanese Aerospace Exploration Agency)

The mission baseline foresees the launch of a composite including both scientific spacecraft, a propulsion module (Mercury Transfer Module or MTM) and a MMO sunshield structure (MOSIF) by Ariane 5 rocket from Kourou (French Guyana) station. The launch is followed by a long cruise phase including gravity assist manoeuvers by Earth, Venus and Mercury. At the end of this long cruise phase, the propulsion module is jettisoned and the composite spacecraft captured to Mercury orbit by weak stability boundary navigation techniques. After delivery of the MMO onto its operational orbit and jettisoning of the MOSIF, the MPO finally reaches its target orbit and starts its scientific mission.

The BepiColombo instruments are conceived and built by Scientific Institutes and National Agencies. The MPO payload complement includes 11 instrument packages: a camera suite with high resolution, stereo, visual and near infrared sensors (SIMBIO-SYS); a laser altimeter (BELA); a set of spectrometers in infrared, ultraviolet, X-ray, gamma-ray and neutron (MERTIS, PHEBUS, MIXS, SIXS, MGNS); a magnetometer (MERMAG); a particle suite (SERENA); and a radio-science experiment composed of an accelerometer and a Ka-band transponder (ISA, MORE). The MMO payload complement includes 5 instrument packages designed to study fields, waves and particles in the Mercury environment: a magnetometer (MERMAG-M/MGF); plasma particle and plasma wave experiments (MPPE, PWI); a sodium atmospheric spectral imager (MSASI); and a dust monitor (MDM).

The Ariane 5 will be used to launch BepiColombo from Kourou. The launch slot opens on the 05 October 2018 and will remain open until 29 November 2018. This is a night-time launch opportunity. There shall be one opportunity every day while the slot is open, i.e. 30 opportunities in total. Each launch opportunity is instantaneous. Launcher injection errors will be absorbed by the first electric propulsion arc, i.e. no launcher injection correction manoeuver using chemical propulsion is required shortly after launch. During the interplanetary cruise, the spacecraft will be given gravity assists by the Earth, Venus and Mercury. During some of the cruise arcs between swing-byes, the spacecraft will be using Electric Propulsion thrusters mounted on the MTM. Trajectory correction manoeuvers required around the swing-byes will be performed using chemical propulsion thrusters mounted on the MTM. After the last Mercury swing-by which propels the spacecraft in the required orbital plane with a relative velocity reduced to $1.85 \mathrm{~km} / \mathrm{s}$, five thrust arcs further reduce the velocity so that the spacecraft is weakly captured by Mercury on the 05 December 2025 . Before capture, the MTM will be jettisoned. The capture will be followed by a sequence of manoeuvers performed with MPO chemical propulsion to stabilize the orbit, and insert the two BepiColombo spacecraft in their respective orbits.

\section{MERTIS INSTRUMENT}

The MPO is a three-axis-stabilized and nadir-pointing spacecraft designed for an operational lifetime of one Earth year (with a possible extension of one year). The MPO will be placed in an inertially fixed polar orbit; the spacecraft has one axis aligned with the nadir direction to allow continuous nadir observation of the planet. The MPO's 2.3-hour loweccentricity orbit will provide excellent spatial resolution over the entire planet's surface. The MPO science payload will investigate Mercury's interior, surface composition and morphology, intrinsic magnetic field, the composition of the exosphere and the coupling between all of these aspects.

The Mercury Radiometer and Thermal Imaging Spectrometer (MERTIS) is one of the science instruments on-board the MPO spacecraft. MERTIS features two channels, the spectrometric (Thermal Infrared Spectrometer, TIS) and the radiometric (Thermal Infrared Radiometer, TIR) channel. Both channels are highly integrated and use the same entrance 
optics and the same calibration sources allowing an in-plane separation arrangement. The highly miniaturized radiometer is positioned at the slit of the spectrometer. Data is obtained in a push-broom mode. While the first channel is used for cross calibration with the spectrometric channel, the latter will allow measurements of the surface temperature down to $100 \mathrm{~K}$ with accuracy better than $1 \mathrm{~K}[2,3,4,5,6,7]$.

MERTIS has heritage from the Mars Express Planetary Fourier Spectrometer (PFS), the Visual and Thermal Imaging Spectrometer (VIRTIS) for the Rosetta and Venus Express missions, and the Bispectral Infrared Detection (BIRD). Due to the integrated instrument approach of the proposed design, MERTIS-TIS and MERTIS-TIR share the same optics, instrument electronics and in-flight calibration components.

As with any other space instrument, the design of the imaging spectrometer MERTIS is driven by the need for miniaturization and low-energy consumption. The design is a modular concept of the sensor head, the electronic units and the power/calibration systems. MERTIS has a mass budget of $3.3 \mathrm{~kg}$ and has an average power consumption of 11 W. MERTISTIS covers the wavelength region of 7-14 mm with 78 spectral channels and a spectral resolution of 1/Dl=78-156, MERTIS-TIR covers the wavelength region of 7-40 mm with 2 spectral channels. Depending on the actual surface properties, the spectral resolution of MERTIS can be adapted to optimize the S/N ratio. This allows us to resolve weak spectral bands from the regolith with $<1 \%$ contrast. MERTIS-TIS will globally map Mercury with a spatial resolution of $500 \mathrm{~m}$ and will map 5-10\% of the surface with a spatial resolution better than $500 \mathrm{~m}$ [2, 4, and 7]. Dimensions of the instrument are approximately $180 \times 180 \times 130 \mathrm{~mm}^{3}$. The external baffles are $200 \mathrm{and} 90 \mathrm{~mm}$ long, the diameter is $75 \mathrm{~mm}$ (see Figure 1).

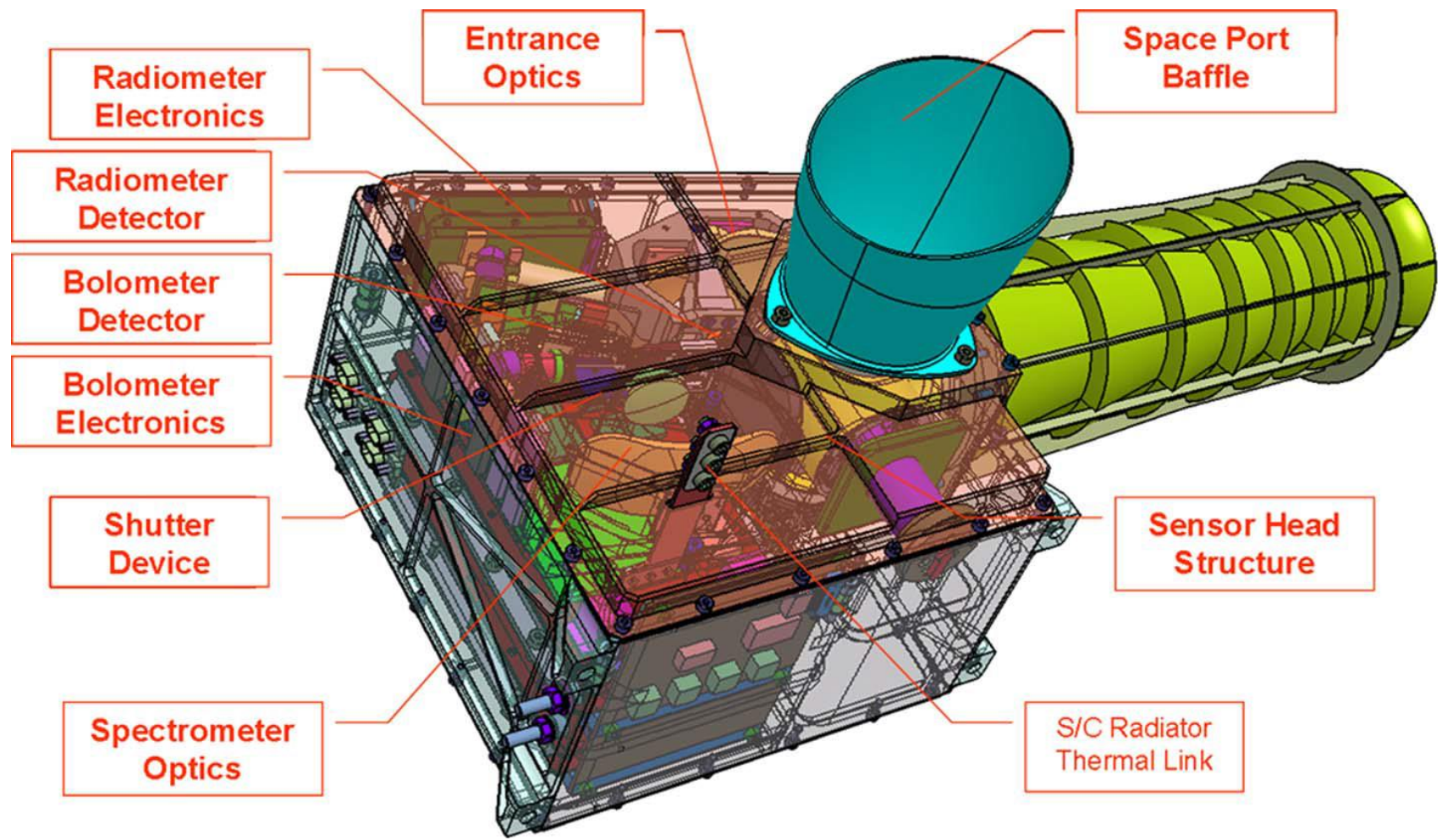

Figure 1. Schematic view of the MERTIS instrument. Clearly visible is the planet baffle (yellowish-greenish), the space port baffle (bluish-greenish) and the housing structure.

\section{MERTIS SCIENCE OPERATIONS PLAN}

This chapter describes the operation of MERTIS in-orbit and during the cruise phase. It describes the general philosophy and the plan of the operations and provides example for different seasonal scenarios. 
MERTIS (MErcury Radiometer and Thermal Infrared Spectrometer) is an imaging infrared spectrometer with an integrated micro-radiometer experiment, and it is located on the BepiColombo Mercury Planetary Orbiter (MPO).

MERTIS has been selected by the BepiColombo Science Programme Committee referring to the R-IMS instrument of the MPO Payload Study Document. Since MERTIS is approved as the only thermal infrared spectrometer of the MPO payload the name of the experiment is MERTIS for the MERcury Thermal Infrared Spectrometer.

Planning the observations for an instrument onboard a remote sensing mission is a fairly complicated goal, and containing itself several different tasks. MERTIS geometric parameters have been documented before launch (mounting, alignment pointing) with references to relevant information and reports, and maintain deviations in-flight. Once integrated on the spacecraft, a set of tests on alignment measurements have been performed, to understand for instrument relevant information, and deduce any parameters and information necessary for the SPICE instrument kernel (IK). SPICE is an observation geometry information system developed from NASA to assist scientists in planning and interpreting scientific observations from space-based instruments onboard robotic spacecraft. The MERTIS IK was developed, updated, and validated (together with other relevant SPICE kernels) with suitable test scenarios.

Our group in Berlin took part to the SGS (Science Ground Segment) system testing and validation, supporting SGS for simulations (i.e. exercise procedures and interactions through the execution of the Operational Rehearsals), and for endto-end testing activities.

Concerning the MERTIS science operations, our activity covers the following areas:

- Support the definition and creation of instrument and science observation models.

- Define observation strategies and scenarios, and identify associated constraints and conditions.

- Define the instrument in-flight pointing and calibration requirements.

- Support the definition of the instrument downlink strategy.

- Support the development of the MERTIS IPOD and review relevant updates to the document.

- Support the assessment of spacecraft capabilities to ensure that the instrument scientific objectives are fulfilled and the performance is not affected (e.g. pointing performance, attitude constraints, orbit evolution, power and thermal envelopes, on-board storage and downlink capabilities and strategies).

- Support the preparation and running of relevant Science Operations Analysis (SOA).

- Pre-launch this analysis foresees the update of the Power and Data modelling, the inclusion of the Pointing analysis for calibration and the assessment of thermal operational constraints.

- Review the MERTIS Science Operations Modeling Analysis (SOMA) resulting from the relevant SOA.

- Support to science operations feasibility analysis during cruise.

The IPOD is a document containing all information about the instrument science operations and in-flight calibrations.

The Science Activity Plan (SAP) for BepiColombo describes in a structured way all scientific activities to be carried out by the instruments on board the Mercury Planetary Orbiter (MPO) throughout the nominal science mission (Dec 2025 to Dec 2026) in order to fulfil the science requirements of the Mission.

The SAP is based on operations requirements of the instrument teams taking the available resources and spacecraft constrains into account. The SAP is under the responsibility of the Project Scientist and supported by the SGS, which performs the analysis of science operations scenarios and proposes an "optimized" operations plan. MERTIS observations are defined in the Observation Catalogue, a web tool made available from ESA for the BepiColombo mission.

Figure 2 shows MERTIS alignment and orientation on the BepiColombo spacecraft. 


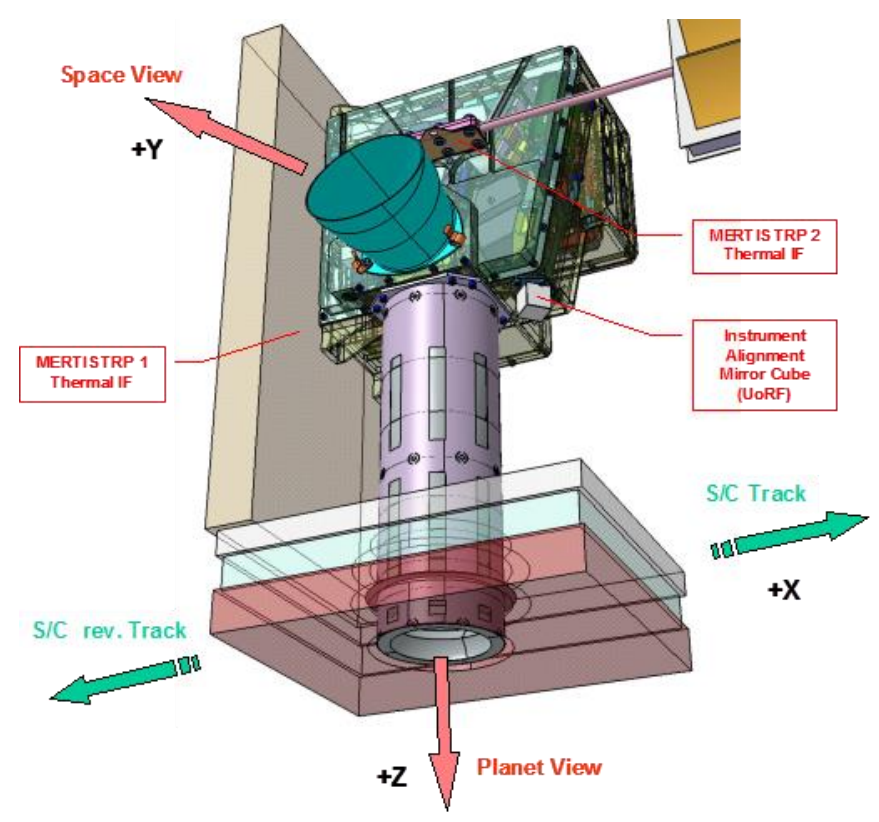

Figure 2. MERTIS alignment and orientation on the BepiColombo spacecraft.

\subsection{Near-Earth Commissioning Phase (NECP)}

During the Near-Earth Commissioning phase, no science observation is done by MERTIS. The only goal is to verify the instrument function and science performance by using the in-flight calibration devices and verify their performance.

Two modes are performed for MERTIS after switching-on:

1. a nominal science mode for several hours

2. the diagnose mode to verify the sub-system functions of MERTIS. During this test, several operation modes for MERTIS will be executed (approx. 30 minutes each) to test the instrument response.

\subsection{Instrument Check-out Plan during Cruise}

The check-out procedure is done once every 6 month during the Cruise phase. The tests to be performed are equal to the tests performed during the Near-Earth Commissioning phase.

\subsection{Near-Mercury Commissioning Phase}

Commissioning near Mercury consists of two phases, starting with the verification of the instrument functionality and basic instrument performance. This is done with the same operations as for the Near-Earth commissioning using the inflight calibration devices and verifying their performance.

Two modes are performed after switching-on:

3. a nominal science mode for several hours

4. the diagnose mode to verify the sub-system functions of MERTIS. During this test, several operation modes for MERTIS will be executed (approx. 30 minutes each) to test the instrument response.

Following this two point, a full operational verification of the instrument performance will be executed. The BepiColombo spacecraft will enter orbit in the Mercury summer season (see Figure 3). After verification of the functionality and basic performance, MERTIS will perform a sequence of observations for several orbits following the general procedures for the survey mapping (see paragraph 3.5.2). This sequence provides data to fully assess the 
operational performance of the instrument. At least $50 \%$ of the test orbits should be consecutive to assess orbit-to-orbit variations.

MERTIS requests these observations as early as possible in the Near-Mercury commissioning phase to cover a significant portion of the surface of Mercury during the summer season. This will allow assessing the signal return from the planet and is essential to optimize if necessary operational parameter for the first high resolution mapping phase in the following winter season.

MERTIS would benefit from ride-along observations with other instruments performing of planet and star calibrations for instrument cross-calibration especially with the SYMBIO-SYS suite.

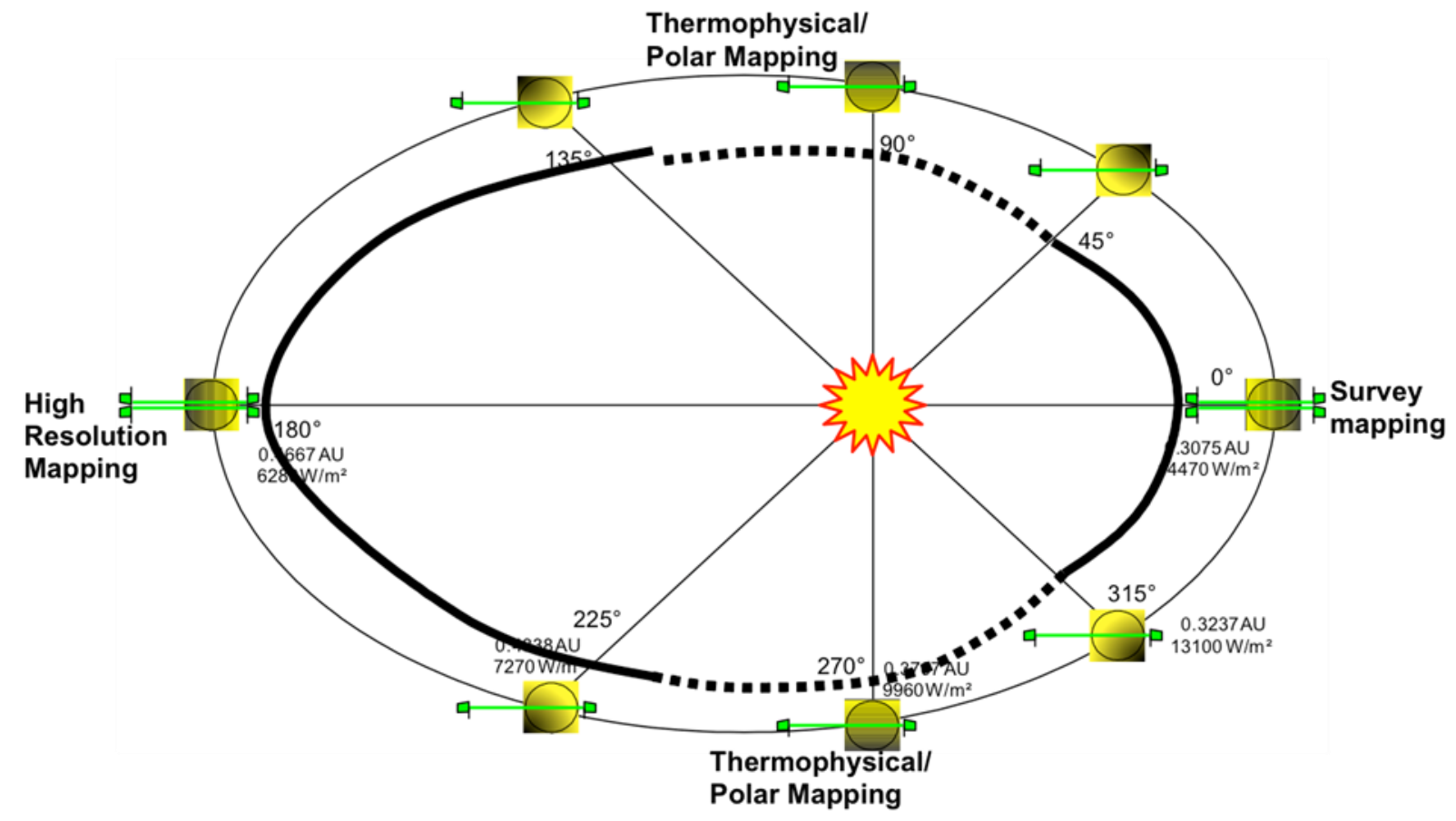

Figure 3. MERTIS operations during a Mercury year.

\subsection{Planetary Fly-By}

Currently observations during the planetary (Venus and Mercury) and lunar fly-by are foreseen and already implemented as option in the MERTIS observations plan. From the scientific point of view it is highly valuable to obtain data during the planetary fly-byes. Especially the lunar fly-by would be of large interest, as there have been numerous comparisons between surface composition of the Moon and Mercury in the literature, and no thermal infrared spectrometer ever took remote sensing measurements in orbit around the Moon. For this reason we characterize a planetary fly-by mode. In this mode the calibrations sequences would be obtained before and after the fly-by. During the fly-by only science frames are obtained. The macropixel parameters and the repetition times are defined based on limitations set by the spacecraft resources, to reduce data volume and power consumption. Data obtained during the lunar fly-by will be used to obtain cross calibration with other instruments and to characterize the inflight behaviour of MERTIS prior to the arrival at Mercury.

The coverage of Venus in the thermal infrared spectral region is very limited. Actual JAXA mission Akatsuki can conduct only broadband observations in the 8-12 $\mu \mathrm{m}$ region, missing most of the $\mathrm{CO}_{2}$ band cantered around $15 \mu \mathrm{m}$. Akatsuki cannot do spectroscopic observations and therefore lacks the capacity of probing several atmospheric depths simultaneously. Also, Akatsuki's view of the planet changes within the orbit and from orbit to orbit, which complicates a homogeneous study of the whole-disk brightness of the planet. The PFS instrument on ESA Venus Express mission unfortunately never returned Venus data, also the only comparable dataset was obtained by a spectrometer on the Russian mission Venera 15 in 1983. MERTIS spectroscopic observations of Venus will allow probing several 
atmospheric depths simultaneously. While the planet view is blocked by the Mercury Transfer Module (MTM), we can still observe Venus using the deep space view. We will perform simultaneous observation with the spectrometer and the radiometer channel. Internal 300K (passive) Blackbody will be used for calibration. Science mode (binning and dwell time) are optimized to maximise the S/N ratio. During Venus Fly-by 1 and 2 we will take acquisitions of Venus for more than 60 minutes using various science modes. Flyby 3 will be used to observe Venus as an exoplanet. Observations of solar system planets, and in particular of their whole-disk signals, provide unique context for the future interpretation of brown dwarf and exoplanet light curves. Observing Venus as an exoplanet will provide insight into the time scales and physics of the phenomena that impact the planet's photometry. This can be achieved if the planet is observed over a long baseline and with an optimal cadence. The baseline is important to identify the longer-time variability (e.g. the occurrence of rotating vortices, large storms, planetary rotation ...). The cadence is of key importance to discern the phenomena (e.g. small cloud structure) that impact the short-time variability. The Venus exoplanet observations can be done when the planet appears sub-pixel in the MERTIS FOV. Figure 4 shows the Fly-byes and opportunities to observe Venus as an exoplanet.

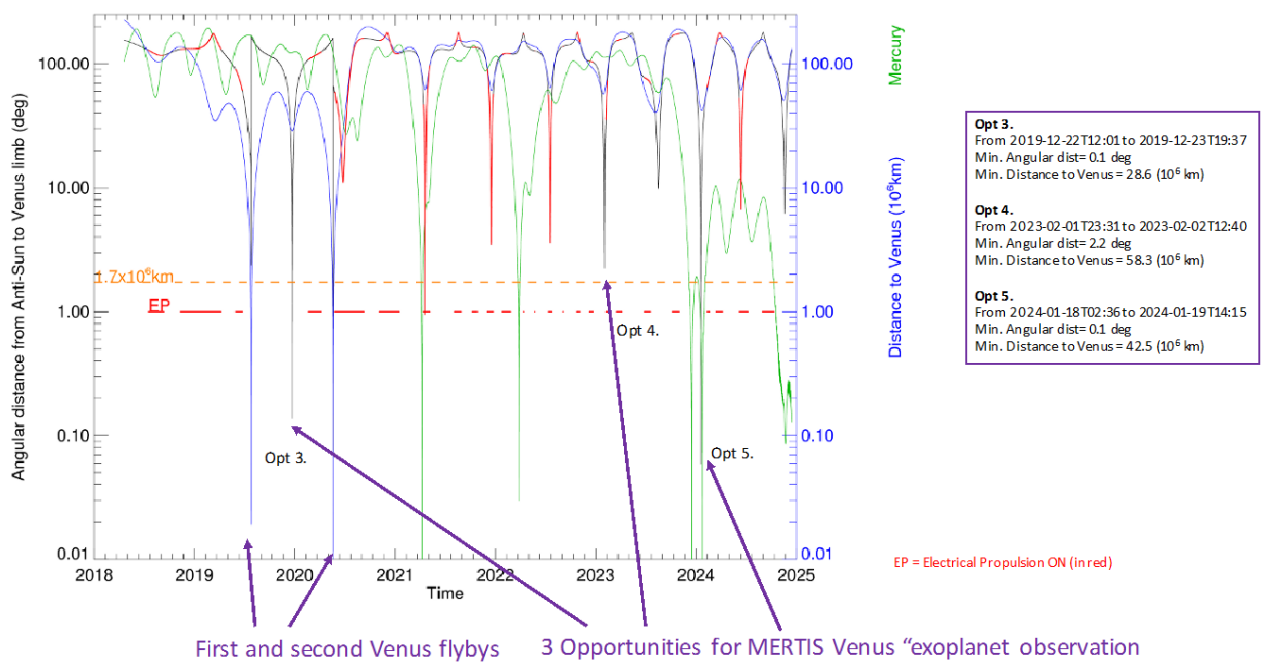

Figure 4. MERTIS observation strategy for Venus Fly-byes.

\subsection{MERTIS Operations in Mercury orbit}

The first science objective of MERTIS is the global mapping of the surface with a spatial resolution of at least $500 \mathrm{~m}$. One of the main scientific goals of MERTIS is to provide a global map of the mineralogy of Mercury at a spatial resolution of at least $500 \mathrm{~m}$. To reach this goal the baseline operation for MERTIS is continuous mapping. The limited resources of the mission make it necessary to optimize the operations of MERTIS so that the global mapping is achieved with minimal overhead. In theory the complete coverage could be achieved after 2 Mercury years. However due to the calibration cycle of MERTIS there are gaps which have to be filled in during the third and fourth Mercury year. The latter have the same illumination conditions as the first two Mercury years. Therefore MERTIS will require the whole mission time to complete the map with highest spatial resolution. In general MERTIS operations follow the seasons of Mercury (see Figure 3).

During the winter season (apohel) the periherm of the spacecraft orbit will be on the dayside. In this period MERTIS will perform high resolution mapping. The data for the global mapping of Mercury at spatial resolutions of at least 500m will 
be obtained during this season. This season gives the best conditions for the mineralogical mapping of the surface, yielding image data with the highest spatial resolution.

During the summer season (perihel) the periherm will be on the nightside. In this period MERTIS will perform survey mapping. This will provide best conditions for the radiometric observations and will allow obtaining temperature mapping with the highest spatial resolution. The dayside perihel passes will yield data with low spatial resolution. Observations by the spectrometer part of MERTIS during this period would mainly be used for planning of the following apohel period. This will allow optimizing the mapping strategy for the remaining part of the mapping phase. This will be especially important directly after the orbit insertion. BepiColombo operations will start in this season and measurements here allow preparing for the first season of high resolution mapping.

The surface temperatures between the summer and winter season will be significantly different. Measuring the same surface area in the both season helps to further constrain thermophysical properties and mineralogy by retrieving information on the temperature dependence of the surface emissivity. In addition the changing viewing conditions between the mapping phases provide further information about the phase dependent surface properties.

During the spring and autumn seasons of Mercury's orbit the orbit of BepiColombo will be close to the terminator. In general these are the least favourable observation conditions for MERTIS. However the strong and rapid change in surface temperature during sunrise will provide a high contrast in the surface radiance. In this period MERTIS will perform thermophysical and polar mapping. In $10 \%$ of the orbits in these seasons MERTIS will obtain data during sunrise and sunset with the spectrometer and the radiometer channel. Under these conditions the surface shows the highest thermal contrasts. These data allow constraining models on the physical properties of the regolith. Furthermore the spring and autumn season provide a good opportunity to study the polar regions of Mercury and mapped the temperature contrasts between permanently shadowed craters and the surrounding plans.

MERTIS requires a track to track overlap of at least $10 \%$ to allow radiometric cross calibration. Due to the characteristics of the orbit and the FOV of MERTIS the overlap is increasing quickly with spacecraft altitude. The large overlap would allow increasing the signal to noise ratio of the instrument. However the limited resources of the mission do not allow downloading the additional data. Therefore the operational scenarios have been optimized to minimize the overlap while guaranteeing the necessary calibration data.

\subsubsection{High resolution mapping}

The MERTIS operations along the orbit are optimized for each season, and to maximize the science return within the limited resources of the spacecraft. MERTIS produces the highest data rates during the high resolution mapping phase. The increasing overlap with spacecraft altitude allows skipping observations during subsequent orbits at higher latitudes without losing coverage as shown in Figure 5. This figure shows the ground track for three consecutive orbits (colour coded in green, orange and blue) in the high resolution mapping phase. Between $46^{\circ} \mathrm{N}$ and S every orbit needs to be observed to avoid gaps in the coverage. However between $30^{\circ}$ and $46^{\circ} \mathrm{N} / \mathrm{S}$ only $70 \%$ in the centre of the detector need to be read-out, which is called a windowing mode. Between $46^{\circ}$ and $58^{\circ} \mathrm{N} / \mathrm{S}$ the ground track of the middle orbit is completely covered by the first and the third orbit. Therefore the MERTIS observations in the middle orbit can be stopped at $46^{\circ} \mathrm{N} / \mathrm{S}$.

Figure 6 shows schematically the sequence for 10 consecutive orbits in the MERTIS observation scheme. Coloured lines indicate that MERTIS is taking data, the dashed line indicate that the windowing mode is used. North and south of $69^{\circ}$ no further optimization is performed to limit the amount of mode switches during an orbit. The sequence repeats after 60 orbits. The data rates given for each latitude range are averaged of the whole sequence. 


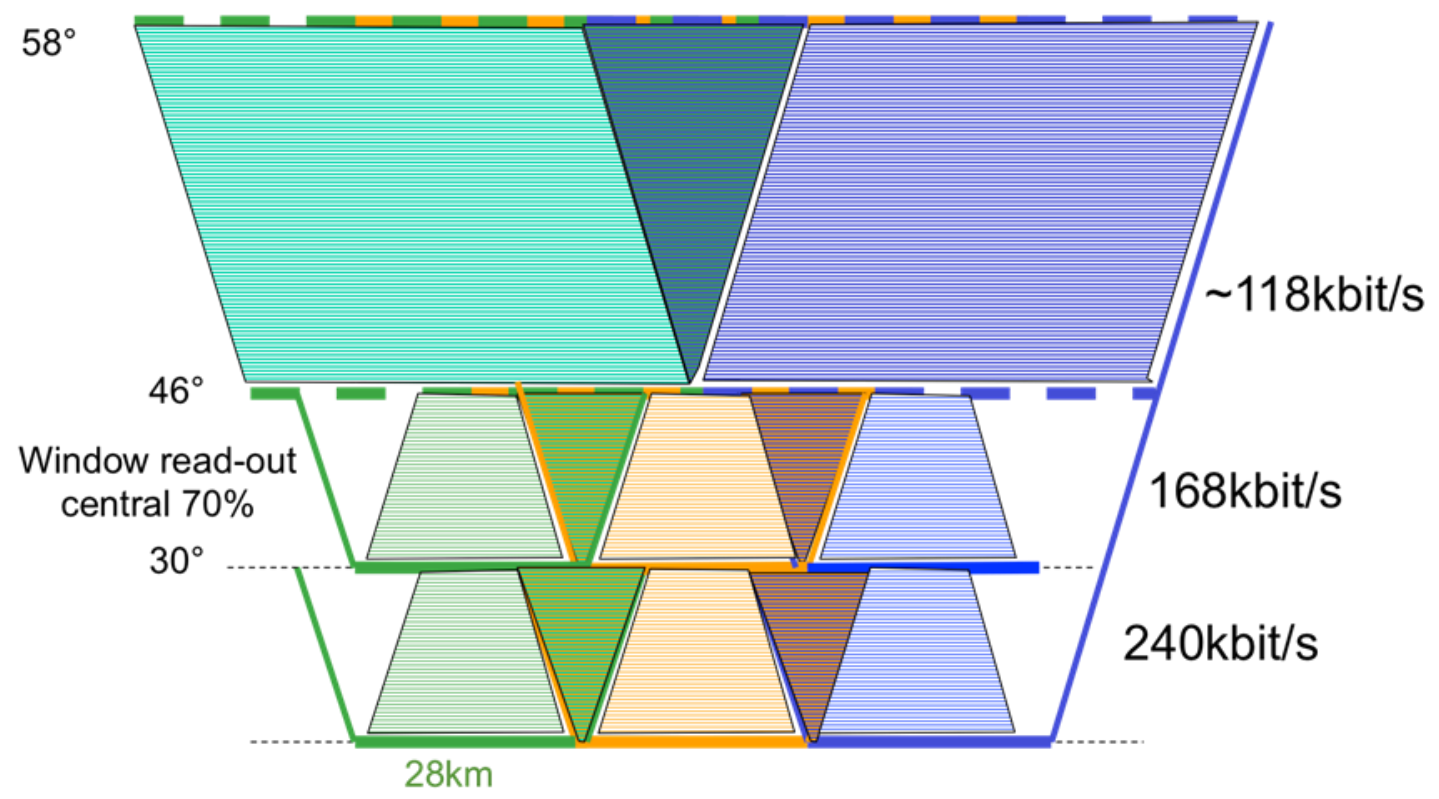

Figure 5. Track coverage for three consecutive orbits in the high resolution mapping phase.

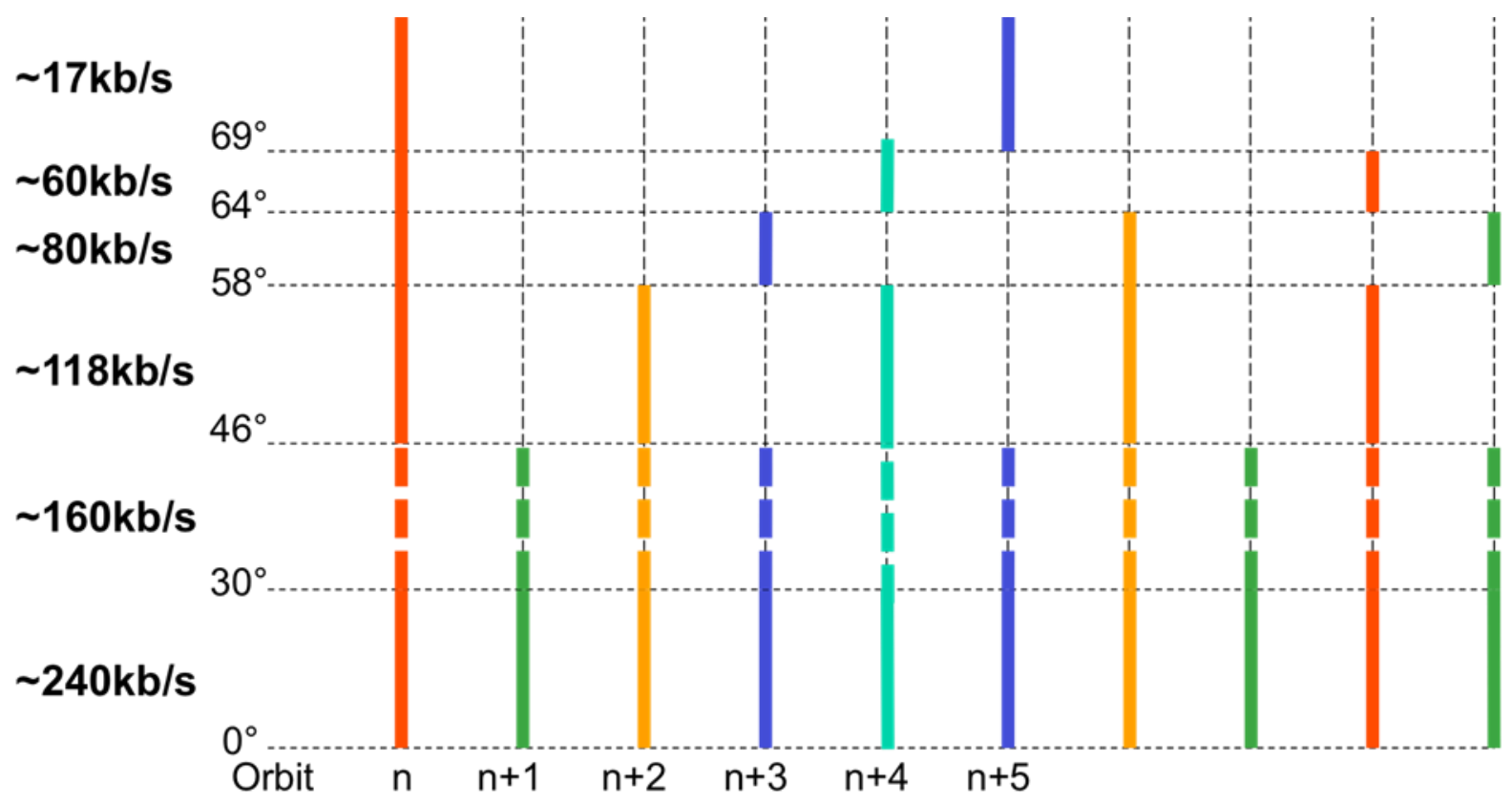

Figure 6. Schematic display of the operational sequence over a period of 10 orbits for MERTIS.

Figure 7 shows the principal operations sequence for one orbit. The shading indicates areas in which either windowing is used or orbits are skipped in order to reduce data rate. The actual sequence during an orbit is depending on the sequence operated on the previous orbit. This approach minimizes the overhead and thereby the total produced data volume significantly, but at the same time increases the complexity of the MERTIS operations significantly. It also increases the risk of loss of coverage in case of anomalous situations on the spacecraft. Depending on the latitude the loss of data from on orbit can affect up to 5 orbits. On the night side of the planet the radiometer is always operated. 


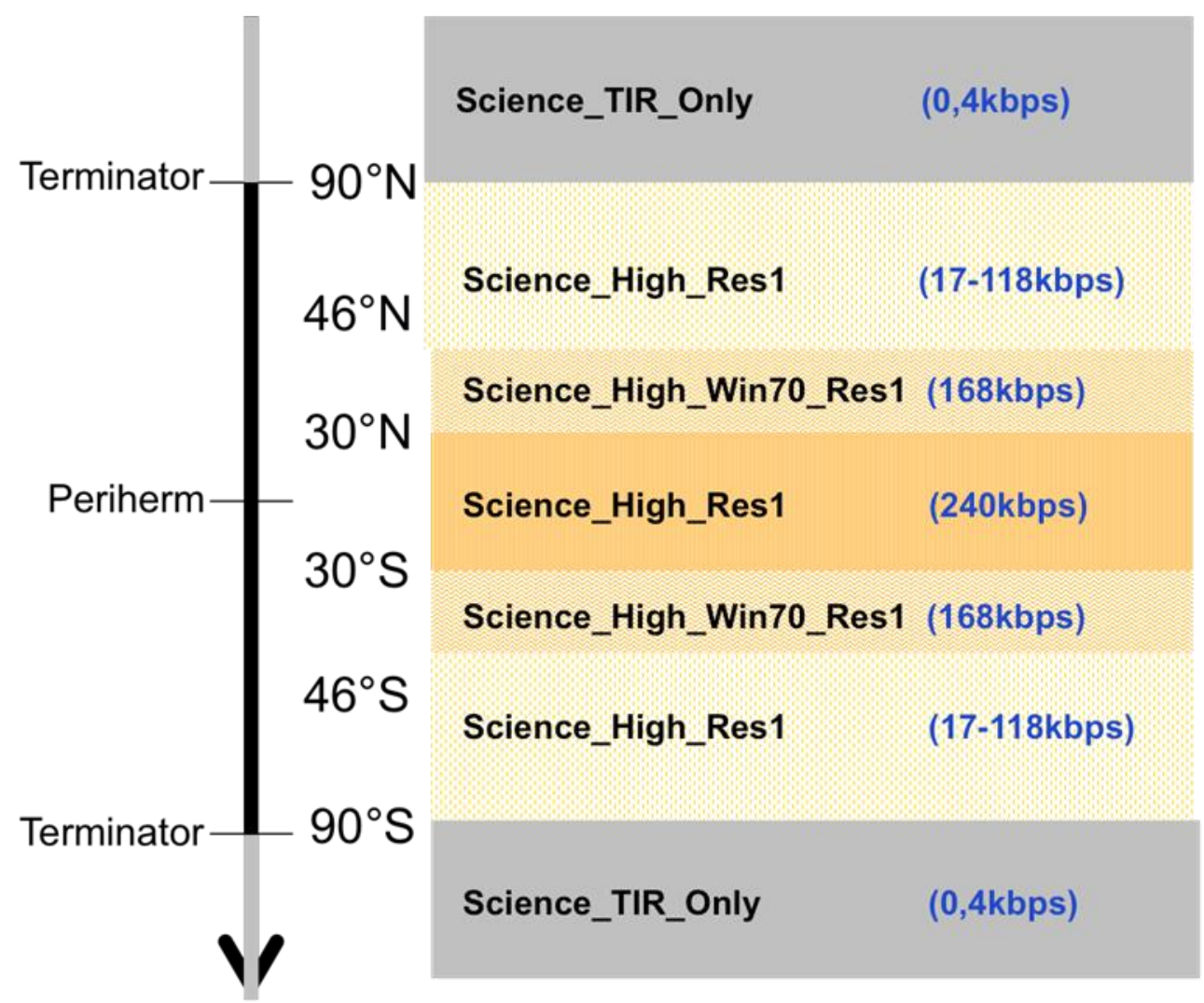

Figure 7. Operational scenario during the high resolution mapping phase with associated data rates.

\subsubsection{Survey mapping}

For the survey mapping a similar sequencing can be applied. The baseline sequence for the survey mode is shown in Figure 8. For this period the largest overlap occurs around the equator and it is reducing towards higher latitudes. For the survey mapping phase MERTIS modes with reduced spatial resolution are used reflecting the larger distance of the spacecraft from the planet during the dayside passage. 


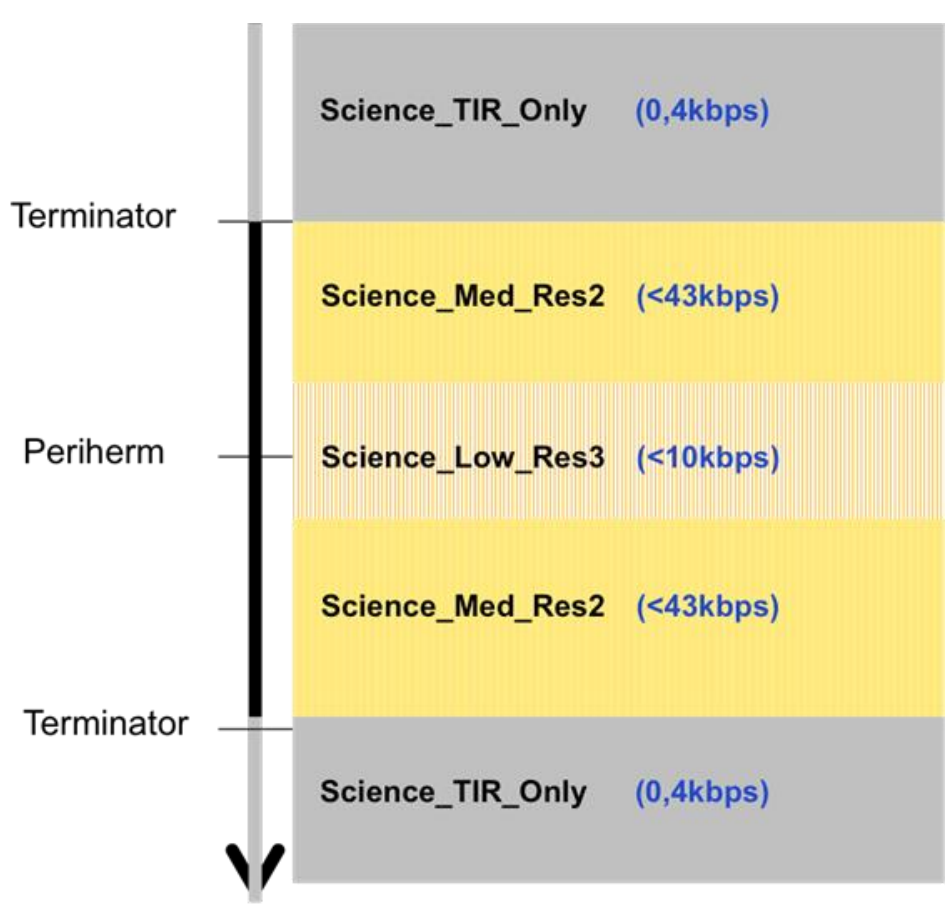

Figure 8. Operational scenario during the survey mapping phase with maximum data rates based on sequencing.

\subsubsection{Thermophysical and polar mapping}

For the thermophysical and polar mapping phase a different approach is used than for the high resolution and the survey mapping modes. The latter two modes are focused on the goal of global mapping. The thermophysical and polar mapping phase takes advantage of the special illumination and observing geometries in the dusk-dawn orbits in the autumn and spring season to obtain observations with maximal thermal contrast. These observations will allow studying for small regions the thermophysical properties of the surface of Mercury in details. In addition this phase can be used to map the polar regions with a good signal-to-noise ratio. The surface temperatures in close to the poles of Mercury are at the sensitivity limit of MERTIS. In order to increase the signal to noise ratio images of consecutive orbits can be stacked if the illumination conditions do not change. Due to the slow rotation rate of Mercury this condition is fulfilled in the spring and autumn seasons. 


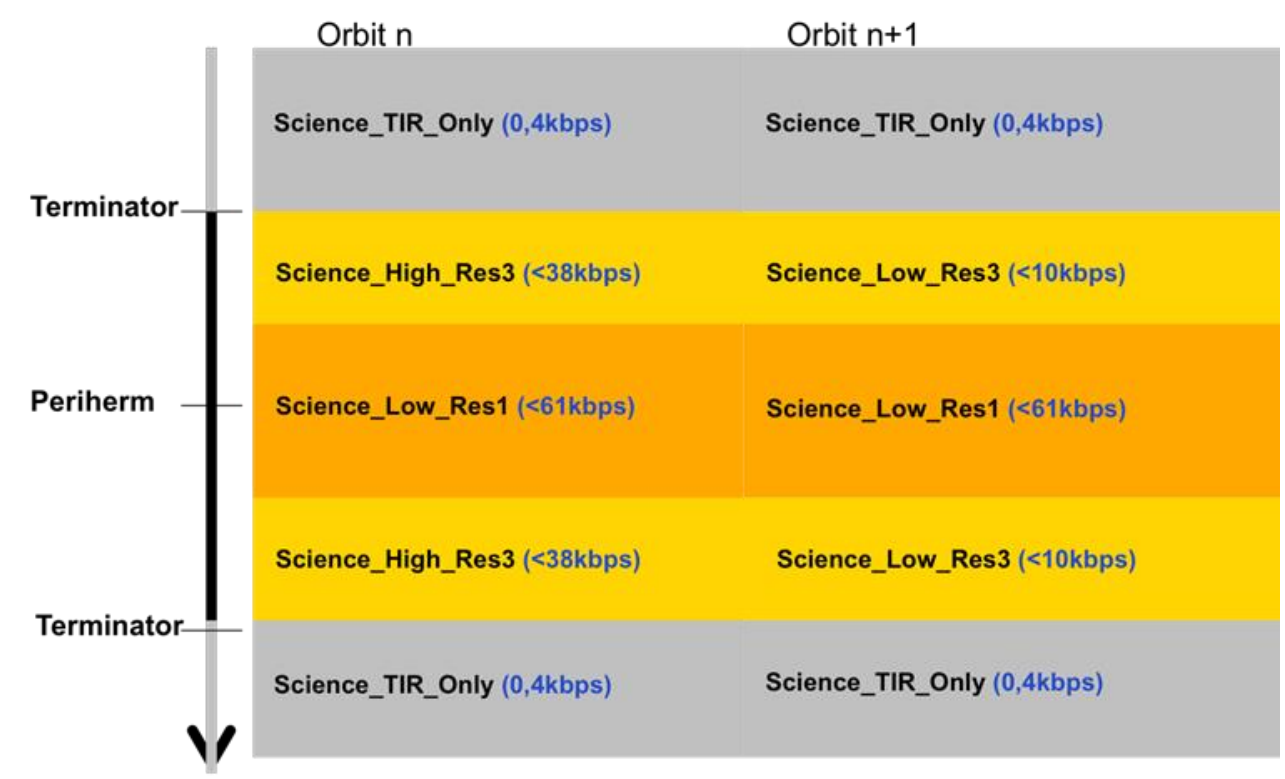

Figure 9. Two orbit sequence in the thermophysical and polar mapping phase.

In the thermophysical and polar mapping phase MERTIS observes only in approx. 10\% of all orbits. The observing sequence during this phase consists of two consecutive orbits. In the polar areas MERTIS alternates between a low and a high resolution mode, to embed high signal-to-noise ratio measurements with high spatial resolution measurements. At low latitudes the low spatial resolution data is obtained to maximise the signal-to-noise ratio for the thermophysical measurements.

\subsubsection{Other modes / Characterization Mode}

MERTIS will operate in the mapping mode mainly during the winter season of Mercury and in radiometric mode during the summer season of Mercury. Additional minor modes can be operated during different seasons of the orbit. The actual setup and importance of each mode is TBC based on a detailed study of the behaviour of the instrument. Among other possible modes, the Characterization Mode is worth spending few details. This is not a scientific mode, but will be used to characterize the instrument functions. During this mode MERTIS will collect mainly calibration frames over an extended period of time. This allows determining the long-time stability of the instrument, identifying possible thermal drifts and used in combination with other instruments it will provide an interference test.

Calibration frames will be obtained with full spectral and spatial resolution. This mode will be used for the commissioning phase and should be used during the operation phase at fixed time intervals to identify long-term effects and possible degradations of the instrument performance. In calibration mode the subsequent frames will be obtained in repetitions not shorter than 2 seconds to reduce the power consumption of the instrument.

\subsection{MERTIS Nominal Operations}

The nominal operation mode during the dayside pass in the mapping mode is continuous push-broom observation with an alternating sequence sciences and calibration frames. The calibration cycle is achieved according to one full rotation of the pointing device and lasts for $20 \mathrm{sec}$. Thus considering the time for approaching every target a full science data cycle will last $13 \mathrm{sec}$ added by 3 calibrations target durations of $1 \mathrm{sec}$ each and start/ stop times of $1 \mathrm{sec}$ in between. The gap caused by the calibration cycle has to be filled by observations on the next orbit or later in the mission. During the periherm passage where the spacecraft has the highest ground speed the calibration cycles can be suspended. The whole 
calibration cycle including the timing is TBC based on detailed modelling and updated performance data for the scanning unit and the detector.

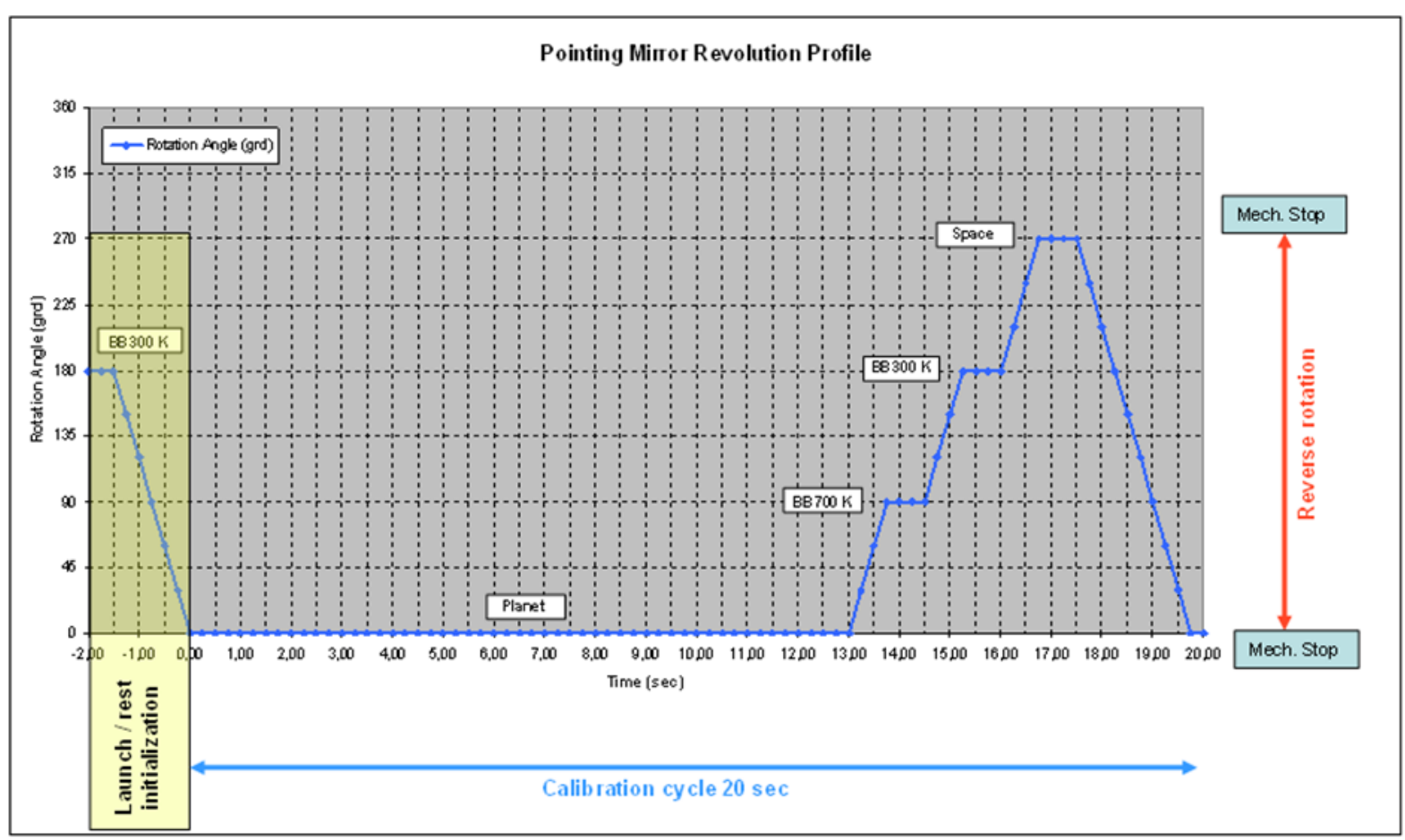

Figure 10. MERTIS calibration cycle (nominal operations).

The nominal operation mode during the nightside pass in the radiometric mode is continuous operation of the radiometric channel of MERTIS with calibration cycles every TBD seconds. For the radiometric observations on the nightside only the $300 \mathrm{~K}$ blackbody and the deep space view are used.

MERTIS can establish macropixel to increase the $\mathrm{S} / \mathrm{N}$ ratio and reduce the data rate. Spectral and spatial directions are treated differently based on the scientific importance of the information. For the spatial direction a simple re-binning is used, which leads to a certain loss of spatial information while increasing the $\mathrm{S} / \mathrm{N}$ ratio and decreasing the data volume. For the spectral direction a moving window average is used, increasing the $\mathrm{S} / \mathrm{N}$ ratio while keeping most of the spectral information.

As macropixels are calculated in software these parameters can be easily adjusted. The baseline for the mapping phase is a latitude dependent binning in spatial direction. The spatial bin size will increase from 1 to 2 moving from the equator to the Polar Regions.

\subsection{In-flight Calibration}

The in-flight calibration strategy will use three calibration sources: two blackbodies, one at $300 \mathrm{~K}$ and one at $700 \mathrm{~K}$ and a view to deep space as a zero radiance measurement. These measurements of calibration targets are performed within the nominal science operation of MERTIS periodically without any special S/C commanding. The period of a calibration cycle is $20 \mathrm{sec}$ as a minimum producing data from the in-flight calibration data with the same spatial and spectral resolution as the science data has.

Nevertheless after each powering-on of MERTIS, a special calibration mode is performed producing a low amount of data but with a maximum of spatial and spectral resolution. 


\section{REFERENCES}

[1] Benkhoff, J., van Casteren, J., Hayakawa, H., Fujimoto, M., Laakso, H., Novara, M., Ferri, P., Middleton, H. R., Ziethe, R., "BepiColombo-Comprehensive exploration of Mercury: Mission overview and science goals," Planetary and Space Science 58, 2-20 (2010).

[2] Hiesinger, H., Helbert, J., and MERTIS Co-I Team, "The Mercury Radiometer and Thermal Infrared Spectrometer (MERTIS) for the BepiColombo mission," Planetary and Space Science 58, 144-165 (2001).

[3] Walter, I., Hirsch, H., Jahn, H., Knollenberg, J., Venus, H., "MERTIS: a highly integrated IR imaging spectrometer," Proc. SPIE 6297, Infrared Spaceborne Remote Sensing XIV, 62970X (8 September 2006); doi: 10.1117/12.679481.

[4] Helbert, J., Hiesinger, H., Walter, I., Säuberlich, T., Maturilli, A., D'Amore, M., Knollenberg, J., Lorenz, E., Peter, G., Arnold, G., "MERTIS: understanding Mercury's surface composition from mid-infrared spectroscopy," Proc. SPIE 7808, Infrared Remote Sensing and Instrumentation XVIII, 78080J (27 August 2010); doi:10.1117/12.859816.

[5] Walter, I., Säuberlich, T., Lieder, M., Rataj, M., Driescher, H., Helbert, J., Hiesinger, H., "Pointing and spectral assignemnt design and control for MERTIS," Proc. SPIE 8867, Infrared Remote Sensing and Instrumentation XXI, 886706 (19 September 2013); doi: 10.1117/12.2023917.

[6] Peter, G., Helbert, J., Hiesinger, H., Weber, I., Walter, I., Arnold, G., Säuberlich, T., "Developing of MERTIS as an advanced process from the study up to the flight model," Proc. SPIE 8867, Infrared Remote Sensing and Instrumentation XXI, 886707 (19 September 2013); doi: 10.1117/12.2024375.

[7] Helbert, H., Hiesinger, H., D'Amore, M., Walter, I., Peter, G., Säuberlich, T., Arnold, G., Maturilli, A., et al., "MERTIS on BepiColombo: seeing Mercury in a new light," Proc. SPIE 8867, Infrared Remote Sensing and Instrumentation XXI, 886705 (19 September 2013); doi: 10.1117/12.2023972. 